\title{
Melt Pool and Microstructure Characterization for AM Model Development
}

\author{
J.T. McKeown ${ }^{1}$, J.D. Roehling ${ }^{1}$, A. Perron ${ }^{1}$, J.-L. Fattebert ${ }^{2}$, J.M.K. Wiezorek ${ }^{3}$, A.J. Clarke ${ }^{4}$, \\ D.J. Bober ${ }^{5}$, J.N. Florando ${ }^{5}$, M. Kumar ${ }^{5}$, S.A. Khairallah ${ }^{6}$ and M.J. Matthews ${ }^{1}$ \\ 1. Materials Science Division, Lawrence Livermore National Laboratory, Livermore, CA, USA. \\ 2. Computational Sciences \& Engineering Division, Oak Ridge National Laboratory, Oak Ridge, TN \\ USA. \\ 3. Department of Mechanical Engineering and Materials Science, University of Pittsburgh, Pittsburgh, \\ PA, USA \\ ${ }^{4 .}$ Metallurgical \& Materials Engineering, Colorado School of Mines, Golden, CO, USA. \\ ${ }^{5}$ Materials Engineering Division, Lawrence Livermore National Laboratory, Livermore, CA, USA. \\ 6. Computational Engineering Division, Lawrence Livermore National Laboratory, Livermore, CA, \\ USA. \\ * Corresponding author: mckeown3@1lnl.gov
}

Additive manufacturing (AM) technologies represent a significant advancement in the ability to create parts with unique geometries and functionalities. However, AM of metals results in parts with large residual stresses and dramatic changes in microstructure compared to conventionally processed materials, leading to significant differences in the constitutive responses of the materials. The development of models that capture the evolution of these complex microstructures and their constitutive response is therefore extremely important. In addition, the evaluation and validation of these models to ensure that they adequately describe AM materials and their performance characteristics is required. Here, we present both in situ and ex situ characterization results of rapid solidification (RS) aimed at developing a mesoscale phase-field model to describe microstructure evolution under RS conditions relevant to metals-based AM. In addition, SEM and TEM microstructure characterization results will be presented that are aimed at development of a microstructurally aware strength model for AM metals.

Microstructure evolution during AM of metals begins in the melt pool, where laser processing involves rapid melting and subsequent rapid solidification (RS) under conditions involving large thermal gradients, high cooling rates, and high solidification front velocities. These conditions present significant challenges for both in situ characterization and modeling efforts. Our in situ characterization work has focused on using time-resolved imaging of solidification fronts with dynamic transmission electron microscopy (DTEM) to capture solid-liquid interface evolution during laser-induced rapid alloy solidification under processing conditions relevant to metals-based AM [1]. RS results will be presented from experiments with Al-based alloys, as shown in Figure 1 for an $\mathrm{Al}-4 \mathrm{at} \% \mathrm{Cu}$ alloy, and $\mathrm{Ni}-\mathrm{Cu}$ alloys. Complementary ex situ solidification and characterization results involving single-track laser melting experiments with Ti$\mathrm{Nb}$ alloys will also be shown. These in situ [2] and ex situ [3] results have been used to benchmark and calibrate a mesoscale phase-field model, in terms of solidification velocity, solid-liquid interface morphology, and non-equilibrium partitioning during RS. This model is coupled to thermodynamic and kinetic databases within the CALPHAD methodology. Phase-field simulations and comparisons with experimental results will be shown.

To begin developing a microstructurally aware strength model, Ti-6Al-4V was chosen as a test case material due to its common use in the AM community and its sensitivity to thermal processing conditions, which can lead to a large variation in the available microstructures. The mechanical response of these 
alloys is complex, and microstructural information is needed to develop this strength model. Results will be presented that examine microstructure in AM Ti-6Al-4V alloys and microstructure evolution upon heat treatment of the AM material, with investigations of length scale, phase fraction, and morphology as a function of processing conditions. Figure 2 shows bright-field images acquired from (a) the AM material and $(b, c)$ two heat treatments to illustrate the changes in microstructure. These results are being used to modify a Zerilli-Armstrong strength model [4,5] to include microstructural and phase-dependent parameters [6].

\section{References:}

[1] JT McKeown et al., JOM 68 (2016), p. 985.

[2] A Perron et al., Modelling Simul. Mater. Sci. Eng. 26 (2017), p. 014002.

[3] JD Roehling et al., JOM 70 (2018), p. 1589.

[4] FJ Zerilli and RW Armstrong, J. Appl. Phys. 61 (1987), p. 1816.

[5] R Mulay et al., Mater. Sci. Eng. A 666 (2016), p. 43.

[6] This work was performed under the auspices of the U.S. Department of Energy by Lawrence Livermore National Laboratory under Contract No. DE-AC52-07NA27344. Portions of this work were funded by the Laboratory Directed Research and Development Program at LLNL under project tracking code 18-SI-003. Research activities at the University of Pittsburgh received support from the National Science Foundation, Division of Materials Research, Metals \& Metallic Nanostructures program through Grants No. DMR 1105757 and DMR 1607922. Research activities at the Colorado School of Mines were supported by Award No. DE-SC0016061 from the U.S. Department of Energy, Office of Basic Energy Sciences, Division of Materials Sciences.

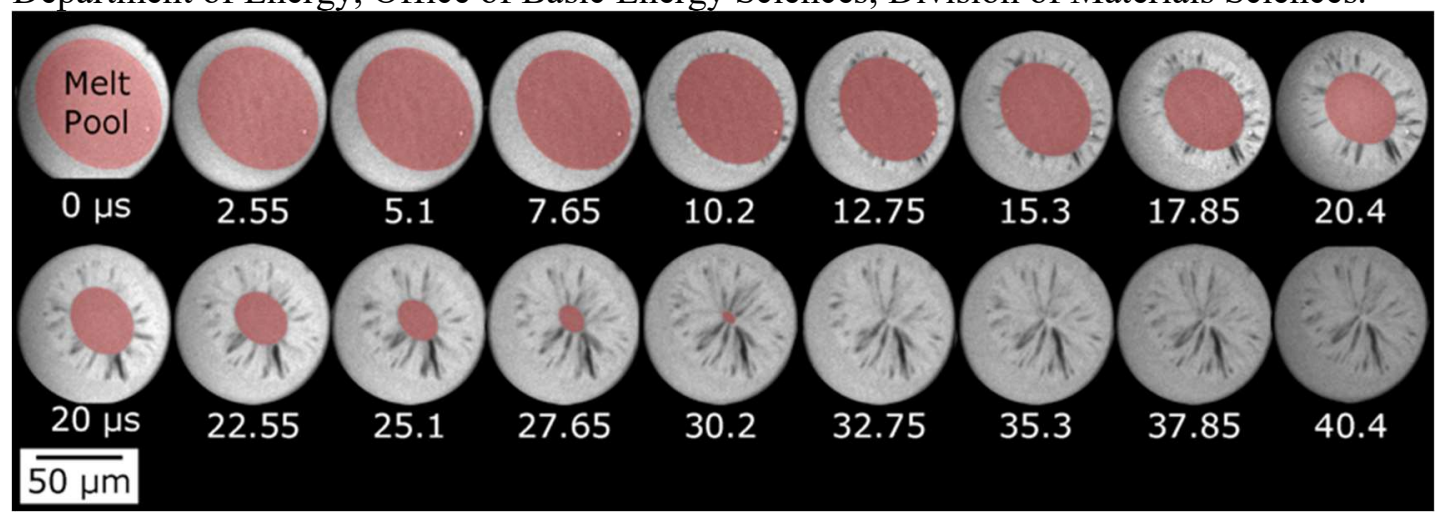

Figure 1. DTEM image sequence acquired during $\mathrm{RS}$ of an $\mathrm{Al}-4 \mathrm{at} \% \mathrm{Cu}$ alloy. Individual images were acquired with $50 \mathrm{~ns}$ electron pulses and $2.5 \mu \mathrm{s}$ between frames. Melt pool false colored for clarity.

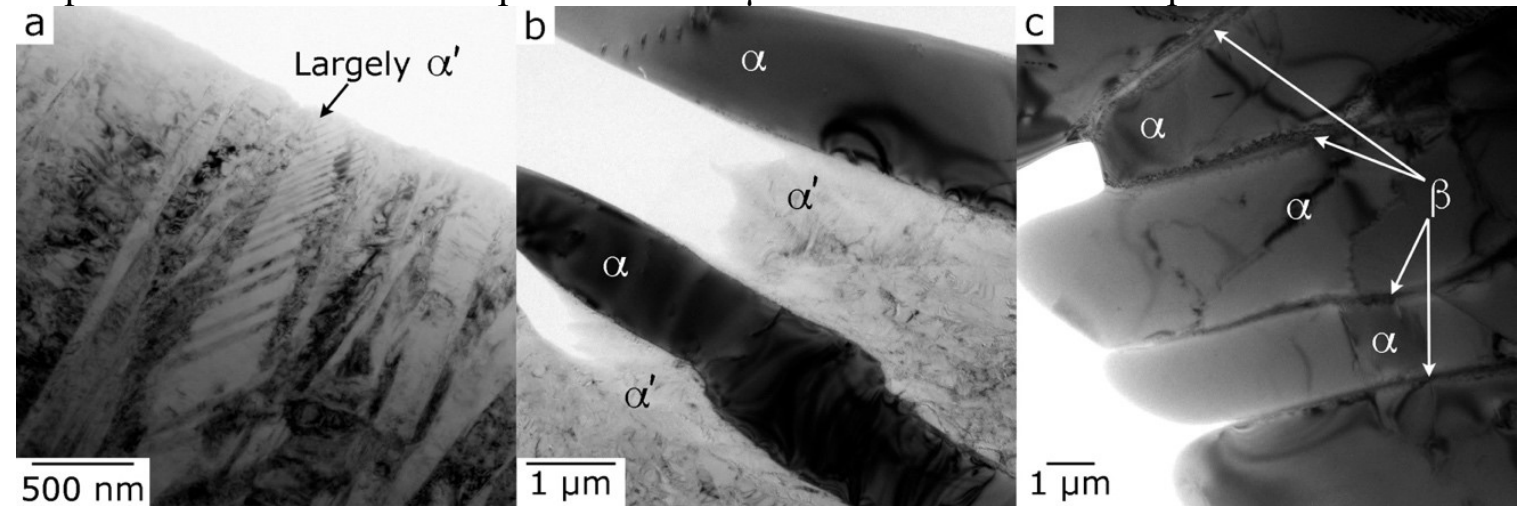

Figure 2. a) AM Ti-6Al-4V, comprised of largely metastable $\alpha^{\prime}$ martensite, b) heat treatment at $950^{\circ} \mathrm{C}$ for $1 \mathrm{~h}$ and water quenching produces an $\alpha-\alpha^{\prime}$ structure, c) heat treatment at $900^{\circ} \mathrm{C}$ for $1 \mathrm{~h}$ and slow cooling yields the typical $\alpha-\beta$ duplex microstructure in Ti-6Al-4V. 